\title{
Aserción, expresión y acción. Una lectura de J.L. Austin
}

TOMÁs ANDRÉs BARRERO

Universidad de los Andes

ta.barrero32@uniandes.edu.co

\begin{abstract}
Resumen: El presente artículo ofrece una interpretación original del trabajo de Austin sobre la aserción y los adverbios y deriva de ella una semántica expresivista para las oraciones de acción. En primer lugar, el análisis austiniano de la aserción con su idea de fuerzas asertivas específicas se conecta sistemáticamente con sus observaciones sobre la modificación adverbial para obtener esquemas de aserción para oraciones de acción. En segundo lugar, se interpretan esos esquemas en términos de la expresión de los compromisos inferenciales propios de prácticas argumentativas exculpatorias, justificativas y ejemplarizantes en las que se explota la contrariedad lógica a la par de la contradicción.
\end{abstract}

Palabras clave: Aserción y oraciones de acción, justificaciones, excusas, contrariedad, incompatibilidad conceptual

\begin{abstract}
This paper offers a new interpretation of John Austin's views both on assertion and on adverbs, as result of which an expressivist thesis concerning the semantics for action sentences is advanced. First, Austin's analysis of assertion based on various, specific assertive forces and his remarks on adverbs are systematically connected in order to obtain assertive schemata for action sentences. Finally, those schemata are put to work as the expression of inferential commitments implicit in argumentative practices of different sorts (exculpatory, justificatory and illustrative) in the deployment of which both logical contrariety and contradiction are exploited.
\end{abstract}

Key words: Assertion and action sentences, justifications, excuses, contrariety, conceptual incompatibility

We become obsessed with "truth" when discussing statements, just as we become obsessed with "freedom" when discussing conduct.

J.L. Austin

\section{Introducción}

La filosofía del lenguaje de J.L. Austin parece dividida en dos compartimentos estancos. El primero incluye todo su trabajo sobre emisiones performativas que ha sido continuado y sistematizado de diversas maneras por filósofos y lingüistas. El segundo contiene los textos en los 
que Austin usa un método que denomina "fenomenología lingüística" y cuyos ejemplos más famosos son los ensayos sobre filosofía de la acción en términos de factores eximentes (Austin 1957) y agravantes (Austin 1966). En este trabajo voy a conectar esos dos compartimentos mediante un estudio sistemático de la aserción para las oraciones de acción.

Desde el punto de vista exegético sostendré que, si se toma en serio el único análisis completo de la aserción en la obra de Austin (Austin 1953), ${ }^{1}$ es posible encontrar una reconstrucción coherente del significado de las oraciones de acción que permita articular la idea de la aserción como acto de habla con los resultados más sugerentes de la fenomenología lingüística para las oraciones de acción. De acuerdo con mi lectura, Austin no se sirve de un solo método filosófico ${ }^{2}$ (el aparentemente casuístico de la "fenomenología lingüística" de los artículos sobre la acción), sino que lo complementa con un análisis lógico-lingüístico sobre la predicación y modificación adverbial guiado por sólidas intuiciones sintácticas.

Mi aporte a la discusión actual sobre la aserción y las oraciones de acción consiste en formular esquemáticamente una semántica expresivista $^{3}$ que pretende caracterizar el concepto de acción a partir de la modificación adverbial, y la modificación adverbial a partir de ciertas prácticas argumentativas. Sostengo que nuestro concepto de acción se articula lógicamente en aserciones, pero las aserciones despliegan

${ }^{1}$ Este texto ha tenido una recepción crítica más bien negativa. Algunos comentaristas de Austin lo han calificado como "un escrito extraño" (Warnock 1989, p. 47), y traductores como Alfonso García Suárez lo han descalificado por ser "un modelo semántico que es un compendio de falta de precaución" que desconoce "los chaparrones fregeanos y posfregeanos" ya caídos en la época en que fue publicado (García Suárez 1975a, p. 25). Sólo Chisholm se atrevió a declararlo "un artículo de la mayor importancia para la filosofía, a pesar de la forma en que está escrito." (Chisholm 1969, p. 104). En la actualidad, Narboux 2011a ha defendido vigorosamente y con sólidos argumentos tanto sistemáticos como histórico-exegéticos la importancia de este trabajo de Austin. De hecho, mi propio trabajo surgió como desarrollo de una sugerencia de Narboux 2011a, p. 193.

${ }^{2}$ Opinión que no es novedosa entre sus comentaristas (Warnock 1989, pp. 2-6), pero sí muy común en algunos de sus críticos (Searle 1969).

${ }^{3}$ Tendencia muy activa y diversa en la actualidad y que puede verse, por ejemplo, en la interpretación expresivista de la lógica de Frege defendida por Brandom 2009 (pp. 179-192), en el expresivismo de Frápolli 2013 con respecto a la verdad o incluso en el expresivismo de Barker 2004 y 2007. El origen de este movimiento es múltiple: viene de Frege, del primer Wittgenstein y su visión de la lógica, de Ramsey y sus ideas sobre la verdad e incluso de la interpretación del lenguaje moral sostenida por los positivistas lógicos.

Diánoia, vol. LX, no. 74 (mayo de 2015). 
su contenido en prácticas agravantes, eximentes o ejemplarizantes, tal como Austin predijo. ${ }^{4}$

En la segunda sección ubicaré el problema de la aserción como parte de una agenda indiscutiblemente fregeana pero desarrollada por Austin bajo su propia adaptación de las categorías de sentido y referencia como dimensiones de evaluación (onus de encaje, dirección de ajuste respectivamente) aplicables al análisis del acto de habla de aserción. Sostendré que, con algo de precaución, esas formas de crítica pueden extenderse a un lenguaje tan marcado por los modificadores adverbiales como el de la acción.

En la tercera sección propongo aplicar los resultados sobre la aserción y los adverbios a una interpretación expresivista del contenido de las oraciones de acción. Cada una de las variedades de aserción mencionadas en la segunda sección tiene una fuerza específica que se hace explícita en el contexto de una práctica argumentativa. Las negaciones desempeñan allí un papel fundamental porque indican unas circunstancias de emisión y evaluación que excluyen o incluyen formas de aplicar el concepto de acción basadas en la contrariedad y no en la contradicción.

\section{Aserción, énfasis y el lenguaje de la acción}

Austin no menciona a Frege en ninguno de sus escritos, ${ }^{5}$ aunque la influencia fregeana es fácilmente detectable en varios puntos clave de su obra. En primer lugar, cuando en How to Do Things With Words (Austin 1975, p. 93) describe el "significado" como "sentido y referencia"; en segundo lugar, en la idea misma de "fuerza" en oposición a "significado" que parece provenir de la idea de "fuerza asertórica" en oposición a "pensamiento" (Frege 1998, p. 203) y, en tercer lugar, en el análisis de

${ }^{4}$ En uno de los pocos lugares en los que se ocupa del asunto, Austin 1940 (p. 57) distingue dos etapas para poder tratar la pregunta por el significado de una palabra. La primera ("sintáctica") consiste en explicar "en palabras" el significado de una determinada expresión intentando definirla y mostrando ejemplos de oraciones en las que aparece. La segunda ("semántica") se ocupa de imaginar situaciones de habla posibles en las que la expresión pueda ser utilizada. Mi objetivo en términos austinianos es entonces desarrollar una "semántica de oraciones de acción" a partir de la correspondiente "sintaxis". Agradezco a una de las evaluaciones anónimas por haber señalado las dificultades inherentes a hablar de una semántica de oraciones de acción en el contexto de la obra de Austin.

${ }^{5}$ Sabemos, sin embargo, que fue el primer traductor al inglés de Los fundamentos de la aritmética y que la traducción se realizó por la misma época en que redactó "How to Talk". 
los enunciados que carecen de referente en términos de presuposición y en su consecuente clasificación como "actos vacíos" (Austin 1975b, Conferencias IV y XI).

Me concentraré en el segundo indicio y mostraré cómo la forma austiniana de tratar la aserción es un desarrollo natural, aunque no fregeano, de un problema ya planteado en "El pensamiento", a saber, el de la distinción entre pensar, juzgar y aseverar (Frege 1998, pp. 202-203). Lo primero corresponde a captar un pensamiento (entidad no mental), lo segundo a un acto de reconocimiento de su verdad, un "proceso mental" como lo llama Frege en otra parte (Frege 1998, p. 239) y lo tercero a una manifestación de ese proceso mental (un acto lingüístico con el que expresamos el juicio). Distinguimos pensar de juzgar formulando una pregunta tipo sí/no que nos permita captar el pensamiento sin reconocerlo como verdadero o falso y sin manifestar ese reconocimiento. La expresión o manifestación del reconocimiento reside en parte "en la forma de la oración asertórica" según Frege.

Pero la fuerza asertórica no depende sólo de la forma asertórica de la oración, como puede mostrarse con el ejemplo de emisiones no serias:

Así como el tronar en el teatro es solamente tronar aparente y la lucha en el teatro es lucha aparente, así también la aserción en el teatro es solamente aserción aparente. [...] En la ficción tenemos el caso de pensamientos que se expresan sin que, a pesar de la forma asertórica, sean realmente propuestos como verdaderos, aunque se pueda sugerir al oyente que él mismo debe dar un juicio aprobatorio. Por tanto, también cuando algo se presente bajo la forma de una oración asertórica, ha de preguntarse siempre si contiene realmente una aserción. Y esta pregunta ha de responderse negativamente cuando falta la necesaria seriedad. (Frege 1998, p. 203)

La fuerza asertórica está subdeterminada por la forma de la oración. El escenario y el teatro aparecen en el análisis de la aserción tan vívidamente como la forma lógica asertiva. Esta idea guía buena parte de la propuesta austiniana en un aspecto fundamental: la importancia de la situación de habla en la determinación de la fuerza ilocucionaria asertiva (Austin 1975, p. 151). Pero hay algo más que simple influencia. Austin adapta la propuesta fregeana de fuerza y contenido a las nuevas exigencias de una filosofía del lenguaje que no hacen de la lógica, y sí del lenguaje ordinario, la herramienta metodológica para tratar el problema de la aserción. El primer cambio se presenta con respecto al célebre "principio de contexto", el segundo con respecto a la relación entre pensamiento expresado y preguntas de tipo sí/no. Ambos tienen consecuencias profundas para la aserción. 
En primer lugar, ya no debemos buscar el significado de la palabra en el contexto de la oración declarativa, sino en el contexto de la oración tal como se usa en la situación de habla para aseverar algo. ${ }^{6}$ La situación de habla no es el estado de cosas que describimos o sobre el cual "informamos" con la emisión, sino las circunstancias de emisión que "indicamos" con ella (Austin 1975, p. 3). ${ }^{7}$ En el lenguaje de la acción, sostendré en 2.2, las situaciones de habla indicadas son aquellas en que nos excusamos o aquellas en que justificamos lo que hicimos, y eso determina variedades de aserción diferentes, como mostraré en 3.2.

En segundo lugar, la expresión de pensamientos no responde necesariamente a preguntas de tipo sí/no. Preguntas como "¿cuál?", “¿cómo?” también son relevantes lógicamente en una situación de habla como veremos en 2.1 y 2.2. La aplicación de este resultado en el lenguaje de acción, argüiré en 2.2 , puede ayudarnos a comprender por qué muchos modificadores adverbiales resultan inapropiados si se busca respuesta a preguntas de tipo sí/no a preguntas planteadas por una intención. En 2.2 voy a desarrollar estas dos novedades a partir de ejemplos de situaciones de habla para el lenguaje de la acción extendiéndolos al modelo de aserción de (Austin 1953) ${ }^{8}$ pero antes de embarcarme en ese proyecto creo necesario hacer ciertas precisiones con respecto a las

${ }^{6}$ Por esa razón cada vez que en lo que sigue use "cuando afirmamos tal y tal cosa", debe entenderse como "cuando utilizamos la oración 'O' (siempre en comillas) para aseverar que tal y tal".

${ }^{7}$ La similitud con la distinción crucial de Barker (Barker 2004, pp. 11-12) entre "reportar" y "expresar" es obvia; la diferencia también: Austin critica duramente la tesis de que el lenguaje es un vehículo para expresar estados psicológicos de los hablantes (Austin 1975, pp. 9-11).

${ }^{8}$ Los defensores y críticos de la distinción locutivo/ilocutivo (Searle 1973, Strawson 1973, Forguson 1973) parecen no reconocer que "How to Talk" es la pieza que falta en la discusión de los actos locutivos en How to Do Things With Words. Tenerla en cuenta hace que la distinción fuerza/contenido heredada de Frege tienda a desvanecerse en concordancia con propuestas expresivistas como la de Barker, ya mencionada (Barker 2004 y 2007), porque si la especificación del sentido y la referencia para las oraciones de acción siempre se producen en el contexto de una variedad de aserción y si el significado locucionario de una aserción es su contenido (Narboux 2011a, §5), el significado locucionario de las oraciones de acción se estructura en cuatro variedades, las cuatro que corresponden a las cuatro especies de aserción y que determinan cuatro fuerzas o énfasis diferentes, como mostraré en el texto. No cabría hablar de un acto locucionario sin tener en cuenta esas fuerzas ni, como parece haberlo hecho Frege, de un pensamiento sin estructura lógica que se articularía en una notación perspicua. Como espero mostrar en las secciones posteriores, nuestro sutil lenguaje de acción se encarga de esta última tarea. 
principales características de ese modelo y su distinción entre onus de encaje y dirección de ajuste.

\subsection{Variedades de aserción, dirección de ajuste y onus de encaje}

Austin no establece un modelo, sino una serie de modelos de aserción. El primero $\left(\mathrm{S}_{0}\right)$ es más esquemático en la medida en que no incluye un dominio de interpretación complejo y en que se supone que las aserciones son más precisas. El segundo $\left(S_{1}\right)$ incluye un modelo de interpretación más rico y una mayor complejidad en la interpretación. Me ocuparé únicamente de este segundo modelo porque se adapta mejor a la terminología filosófica de la acción. ${ }^{9} \mathrm{~S}_{1}$ no se ocupa de oraciones de acción, sino de oraciones del tipo

"I es un T"

en las que "I" denota una variable para un ítem no lingüístico y "T" una variable para un tipo (Austin 1953, p. 135). Los tipos e ítems sólo se distinguen en virtud de la forma en la que aparecen en las aserciones, es decir, como valores semánticos y no como entidades. Para evitar connotaciones metafísicas, podemos hablar de ellos como patterns (modelos) y samples (muestras), respectivamente (Austin 1953, p. 145).

No es posible entender el significado de dichos términos si no es en el contexto de una determinada variedad de aserción. Veamos, ${ }^{10}$ por ejemplo, la oración:

"Julio César es un general romano".

Hay dos tipos de convenciones que se entrecruzan en su intepretación: convenciones de referencia y convenciones de sentido. Las primeras explican cómo conectamos las expresiones con los objetos no lingüísticos, el nombre propio "Julio César" con el personaje histórico o la expresión "ser un general romano" con, por ejemplo, un determinado grupo de personas. Las segundas ilustran cómo conectamos tipos de ítems (en este caso tipos de personas) con expresiones para tipos (Austin 1953, p. 136).

Con las convenciones de referencia intentamos ajustar (fit) expresiones a objetos o tipos u objetos o tipos a expresiones. Aquello que intentamos ajustar o coincide con el elemento que resulta polémico o

${ }^{9}$ Agradezco a una de las evaluaciones anónimas por haber recalcado estas diferencias entre $S_{0}$ y $S_{1}$.

${ }^{10}$ A diferencia de Austin y para que la exposición resulte sencilla, uso nombres propios en vez de numerales para denotar ítems.

Diánoia, vol. LX, no. 74 (mayo de 2015). 
puede entrar en disputa en una situación de habla. Cuando se produce una determinada aserción, parte de lo que se quiere expresar o indicar con la oración que se asevera es ese elemento que llamaré, siguiendo a Narboux 2011a, el foco de la aserción, y que indicaré en mis ejemplos mediante el uso de cursivas.

Las convenciones de sentido funcionan de otra manera. Con ellas queremos establecer una relación sólo entre tipos de objetos y sentidos de expresiones. Esta relación es siempre general, pues el "objeto" del que hablamos siempre se presenta como miembro de una determinada categoría y no como individuo. Por ejemplo, podemos pensar si "Julio César" es un tipo de expresión que sirve para referirse a personas o si "ser un general romano" es una expresión del tipo correcto para servir como predicado de personas. Lo que buscamos aquí es ver si el sentido de una determinada expresión encaja con el tipo de objetos que sirven como valores semánticos de esa expresión o si el tipo de objetos encaja con el sentido expresado. Estas convenciones de sentido establecen un determinado "onus de encaje" (onus of match).

Todas esas distinciones se marcan en las situaciones de habla que expresamos al utilizar una determinada oración para aseverar algo y, a diferencia de lo que sucede según Frege, muchas de esas aserciones no se reconstruyen naturalmente como la respuesta a una pregunta de tipo sí/no. Veamos los siguientes ejemplos:

As.1. "Julio César es un general romano".

As.2. "Julio César es un general romano".

As.3. "Julio César es un general romano".

As.4. "Julio César es un general romano".

As. 1 cumple con los parámetros fregeanos de la aserción: es la respuesta a la pregunta "¿Es Julio César un general romano?". No se pregunta quién es Julio César, sino si la expresión "ser un general romano" se puede predicar de él. Describimos a Julio César como "general romano" (dirección de ajuste) y afirmamos que es un objeto del tipo adecuado como para encajar con el sentido de la expresión "general romano". As. 1 es falsa si Julio César no es un objeto del que pueda predicarse que es un general romano.

As.2 es una respuesta posible a la pregunta “¿Quién es Julio César?" emitida, por ejemplo, por una persona que no conozca nada acerca de Julio César. Llamamos "Julio César" a este hombre (dirección de ajuste) y afirmamos que el sentido de la expresión "Julio César" en su calidad de nombre de persona encaja con el tipo de expresiones que sirven 
como nombres de persona (onus de encaje). As.2 es falsa si con "Julio César" nos referimos a Julio César Chávez, por ejemplo.

As.3 es una respuesta posible a la pregunta "¿Quién es un general romano?" emitida por alguien que necesite conocer ejemplos de generales romanos famosos. La expresión "ser un general romano" se ajusta a este hombre del que hablamos (dirección de ajuste) y afirmamos que el sentido de tal expresión encaja con el tipo de objetos que son generales romanos (onus de encaje). As. 3 es falsa si Julio César no se considera propiamente un general, sino, digamos, un mercenario.

As.4 es una posible respuesta a la pregunta “¿Cuál de estos hombres es un general romano?" emitida, por ejemplo, en el contexto de un juego en el que tenemos que clasificar a Julio César, representado en un busto, como aquel que en un determinado grupo de personajes históricos se ajusta al epíteto "ser un general romano". Clasificamos este hombre de la estatua como "un general romano" (dirección de ajuste), y afirmamos que es un tipo de objeto que encaja con el sentido de la expresión "un general romano" (onus de encaje). As.4 es falsa si la persona que clasifiqué como general romano resulta no ser Julio César, sino, digamos, un orador bizantino cuyo busto se parece mucho al de Julio César.

Sentido y referencia, entendidos desde el uso, son dimensiones de evaluación a las que responden de manera diferente diversas aserciones. El análisis focal de la predicación que he esbozado trae como consecuencia una complejidad lógica mayor que la que se deriva de la dirección de ajuste de la palabra al mundo, tradicionalmente reconocida como la dirección de ajuste para aserciones, ${ }^{11}$ y ello se debe a que para Austin hay desde el inicio dos tipos de legislación lingüística que funcionan en las aserciones: convenciones de referencia y convenciones de sentido. En la situación simplificada $S_{1}$, Austin distingue cuatro especies en el género de la aserción As.1-4 que corresponden a las posibilidades generadas por las dos dimensiones de evaluación de dirección de ajuste y onus de encaje. Éstas son las categorías que adaptaré al lenguaje de la acción.

\subsection{El lenguaje de la acción}

Muchos filósofos han querido saber qué es una acción, cuáles son las condiciones necesarias y suficientes para poder aplicar correctamente el concepto de acción. Por ejemplo, Davidson 1995 (p. 63) comienza uno

${ }^{11} \mathrm{Tal}$ como la usa Searle en su taxonomía de los actos ilocucionarios (Searle 1979) y en su teoría de la intencionalidad de la mente (Searle 1999).

Diánoia, vol. LX, no. 74 (mayo de 2015). 
de sus artículos más famosos sobre el tema con la siguiente pregunta: "¿Qué sucesos en la vida de una persona revelan actuación; cuáles son sus actos y sus obras, a diferencia de meros aconteceres en su historia; cuál es la marca que distingue sus acciones?"

Aunque las respuestas a esta pregunta varían, la pregunta misma presupone una condición obvia: las acciones son una clase de sucesos; si seguimos a este filósofo (Davidson 1995, p. 289), son la clase de sucesos que pueden describirse en términos de una intención. Determinar esa clase de sucesos requiere entonces encontrar descripciones intencionales adecuadas en términos de creencias y deseos racionalizadores.

Por el contrario, para Austin la pregunta “¿qué es una acción?” debe responderse prestando atención a todo el vocabulario de la acción y no sólo a las descripciones intencionales en términos de creencias y deseos. La pregunta "¿qué suceso es una acción?” carece de sentido hasta que se la coloque en una situación de habla, es decir, hasta que se la interprete en términos de una posible variedad de aserción (no necesariamente descriptiva) con una fuerza asertórica específica según el modelo de 2.1.

El contraste entre Austin y Davidson no se debe solamente a las acostumbradas discusiones sobre causalidad física y mental o racionalización, sino a una comprensión diferente del lenguaje de la acción. Denominaré "indirecta" la tesis lingüística de Austin porque, según ella, la pregunta sobre qué suceso es una acción es lógicamente posterior a la pregunta de qué clase de expresiones utilizamos para calificarlo ${ }^{12}$ (Austin 1957, pp. 191, 199, 200, 201; Austin 1966, p. 282; Austin 1953). La primera pregunta no tiene sentido si no tenemos en mente la segunda.

Lo primero que hay que notar es una diferencia gramatical entre el modelo $\mathrm{S}_{1}$ y las oraciones de acción. Una oración de acción incluye como mínimo un sujeto, un verbo y un objeto directo; llamemos a esta estructura sintáctica "pro-oración de acción" o "esquema de oración de acción":

Acc. $\mathrm{x}$ hizo A. ${ }^{13}$

${ }^{12}$ En este trabajo dejo de lado deliberadamente los adverbios de tiempo y lugar, bien explorados por algunos davidsonianos (Taylor 1985, Lepore y Ludwig 2007), lo que implica dejar de lado las preguntas “icuándo?” y “dónde” en la aserción de las oraciones de acción, dado que son comunes a cualquier variedad de aserción, como ha mostrado Anscombe 1979. Irónicamente, si se tiene en cuenta su influencia sobre Davidson, Anscombe comparte un enfoque indirecto al concepto de acción (Anscombe 1979, p. 232). Para una discusión del contraste entre Davidson y Anscombe, véase Annas 1976.

${ }^{13}$ La primera dificultad se presenta cuando intentamos parafrasear las oraciones 
Muchas de las circunstancias de habla en las que aseveramos una oración de acción marcan un determinado aspecto que no se reconstruye naturalmente como una respuesta a una pregunta de tipo sí/no, tal como en las aserciones de $S_{1}$. De acuerdo con el análisis indirecto del concepto de acción, las expresiones adverbiales, su orden y sus posibles negaciones afectan la aserción porque modifican el contenido que ella expresa. Marquemos esas posiciones sintácticamente admisibles en (Acc.):

Acc.1. 1 él hizo A.

Acc.2. Él 2 hizo A.

Acc.3. Él hizo 3 A.

Acc.4. Él hizo A 4 .

Cada posición puede modificar un elemento indicado por las circunstancias de emisión y codificado en una determinada fuerza asertiva; cada una de ellas enfatiza un sentido posible de la oración de acción que puede ilustrarse con los adverbios de acción. A grandes rasgos, podemos agrupar esas posibilidades en dos. Mientras Acc.1 y Acc.2 fraccionan la acción, Acc. 3 y Acc. 4 la presentan como un todo. ${ }^{14}$ Por ejemplo, llenemos el espacio en blanco con "torpemente" (Austin 1957, p. 199):

i. "Torpemente, él pisó el caracol".

ii. "Él, torpemente, pisó el caracol".

iii. "Él pisó torpemente el caracol".

iv. "Él pisó el caracol torpemente".

Si nos guiamos por su posición más cerca del sujeto que del verbo o el complemento directo, i y ii califican más un aspecto del agente que de la acción; nos señalan que hay algo torpe en su pisar el caracol porque sucede casualmente en el contexto de un plan suyo de hacer otra cosa: no ejecuta correctamente otra acción que no era ni incluía como parte de su ejecución pisar el caracol. La paráfrasis más perspicua en este caso es "fue torpe de su parte pisar el caracol". Por esa razón, en esta posición "torpemente" se interpreta como "un adverbio de frase" (Taylor 1985,

de acción, oraciones en las que aparece "hacer" como "proverbo" (denominación que tomo de Grice 1986, p. 11) en términos de oraciones sobre sucesos, oraciones en las que el proverbo es "ser". Véase Barrero 2013 para una discusión de ese problema.

${ }^{14}$ En Parsons 1990 (cap. 4) hay una presentación de la relación entre la posición de los adverbios y su clasificación semántica. Aunque no comparto todos sus resultados, creo que es una buena base de discusión. 
p. 20) o "un modificador orientado al sujeto" (Parsons 1990, p. 64), y no como un adverbio de modo. Indica situaciones de habla en las que nuestro agente quiere acercarse a otro caminante y en su prisa le da un pisotón al caracol. Podríamos preguntarle "¿Simplemente pisaste el caracol?”, y él debería contestarnos conectando su ejecución física con un plan de acción.

Supongamos ahora que iii y iv se emiten en el contexto de un concurso de pisar caracoles con la punta de los dedos. Ambas oraciones califican la acción de pisar el caracol en términos de un determinado modelo y se interpretan naturalmente como respuestas a la pregunta “¿Cómo hizo él A?”. El adverbio es de modo.

La posición del adverbio de torpeza con respecto al agente o al verbo determina así diferentes interpretaciones posibles que enfocan la aserción en el control del agente o en la apariencia física de su acto. ${ }^{15}$ Siguiendo a Austin 1957 (pp. 143, 176) y la convención de 2.1, voy a marcar esa diferencia mediante el uso de cursivas para el foco de aserción. Dadas las circunstancias de emisión, este elemento en el que se enfoca la aserción resulta debatible o extraño. El pensamiento (no fregeano) expresado se detecta con la pregunta contextualmente relevante. $^{16}$

\subsection{Oraciones de acción, aserción, predicación y énfasis}

En el primer caso (i y ii) señalamos la impropiedad de clasificar sin más su movimiento como su acción de pisar el caracol. Es fortuito, no está incluido en su plan y se produce como consecuencia de su falta de control. Sí, él lo pisó, pero casualmente. En estas situaciones de habla, el sentido de la expresión "pisar el caracol" se da por sentado y se señala la posibilidad de que el tipo al que su movimiento físico pertenece (movimiento corporal incontrolado) no concuerde simple o llanamente con el sentido de la expresión. Discutimos que se pueda aseverar con propiedad que él pisó el caracol. No pretendemos, por así

${ }^{15}$ Austin 1957 (pp. 199-200, 282) subraya que estas dos construcciones en el caso de "deliberadamente" producen una distinción entre el aspecto mental de la acción (i y ii) en que el agente se pregunta si debe o no debe hacer algo y el aspecto físico de realizarse con lentitud (iii y iv). Con "torpemente" nos concentramos en la etapa y los errores de ejecución, no de decisión.

${ }^{16}$ Esto quiere decir que la distinción entre sujeto y predicado en las oraciones de acción depende del contexto, no es absoluta. Hay buenos ejemplos de análisis inspirados por el propio Frege que la pasan por alto. Véase Chateaubriand 2001 (cap. 2).

Diánoia, vol. LX, no. 74 (mayo de 2015). 
decir, mirar directamente en sus movimientos físicos e identificar cuál de ellos es la acción de pisar el caracol.

Con Anscombe 1979 (p. 225) afirmamos que las condiciones de identidad para las acciones son dependen del contexto de aserción y del concepto involucrado en la identificación. Nuestro agente no consigue percibir su movimiento físico de pisar el caracol como perteneciente al tipo de suceso que él clasificaría sin más como su acción de pisar el caracol. Lo que está en disputa es cuál de sus movimientos físicos puede identificarse llanamente con "pisar el caracol" dado el plan que él tenía. Señalo este énfasis con un tipo de predicación en la que el objeto de controversia es que él haya pisado el caracol:

c. "Él pisó el caracol". ${ }^{17}$

Contra Anscombe, no pretendemos reducir todas las aserciones a descripciones. ${ }^{18}$ De hecho, en el segundo caso (iii y iv), el adverbio señala la impropiedad de ejemplificar el tipo pisar el caracol mediante su movimiento físico y, por lo tanto, la expresión "pisó el caracol” cumple con la función predicativa:

e. "Él pisó el caracol".

Cuando aseveramos iii y iv damos por sentado que pisar el caracol es su acción, pero el adverbio señala que ella no se adecua a lo que en este contexto de habla llamaríamos "pisar el caracol"; su acción es torpe como ejemplo de "pisar el caracol". Como veremos en 3.2, con las acciones que incluyen un parámetro social es posible que la lejanía del movimiento físico con respecto al parámetro nos impida ver éste como un ejemplo de aquél. Aquí se da por sentado el tipo (el movimiento físico que es su acción), pero se pregunta si este movimiento físico concuerda o no con el sentido de la expresión "pisar el caracol".

Siguiendo la línea descrita en 2.1 y extendiéndola a estos nuevos ejemplos de predicación, cuando al aseverar una oración de acción intentamos ajustar sucesos a nombres (como en c y e) tenemos la misma

${ }^{17}$ Adverbios como "intencionalmente" y "por accidente" aceptan formalmente las construcciones Acc.1 y Acc.2, pero su interpretación es muy problemática. Parsons 1990 (p. 67) parece sugerir que, dado que "intencionalmente" siempre funciona como un modificador orientado hacia el sujeto, entonces no hay diferencia de sentido entre Acc.1, Acc.2 y Acc.3, Acc. 4. Davidson 1995 (p. 109) simplemente descarta Acc. 1 y Acc. 2 y se concentra en Acc. 4. Encuentro un matiz de arbitrariedad en ambas posiciones. Pospongo la discusión de este problema hasta el fin de esta sección.

${ }^{18}$ Lo que hace justicia a algunos de sus críticos, como Kim, cuando señalan las diferencias entre sucesos genéricos e individuales (Anscombe 1979, p. 231).

Diánoia, vol. LX, no. 74 (mayo de 2015). 
dirección de ajuste. Pero cuando nuestro interés está en ver que él haya pisado el caracol como muestra del modelo social de pisar caracoles, o bien en ver de qué tipo de modelo podría ser muestra su movimiento físico, se hace evidente que hay diferencias en la forma en que encajan el modelo y el sentido. La dirección de ajuste es igual en c y e, pero el onus de encaje es diferente (Narboux 2011a, §4). Podemos clasificar un suceso (pisar el caracol) como una acción en línea con los casos 1 y 2 o bien podemos ejemplificar un tipo de acción (pisar el caracol) mediante un suceso determinado (movimiento corporal), en línea con los casos iii y iv.

Usando otros modificadores adverbiales se hacen explícitas las otras dos especies de aserción, llamar y describir. Por ejemplo si, en consonancia con Acc.4, emitimos v, el agente pudo haberse resbalado en la acera para evitar una motocicleta que lo iba a atropellar y, por azar, pisó el caracol:

v. "Él pisó el caracol por accidente",

En esa situación de habla no llamaríamos "acción" al suceso de pisar el caracol. A diferencia de iv, la posición del modificador adverbial de accidentalidad afecta el verbo y rompe la conexión entre el sujeto y el objeto directo. Es un movimiento de su cuerpo que no podríamos identificar o ubicar como acción suya. "Por accidente" es un "modificador de oraciones" (Taylor 1985, p. 20) que puede parafrasearse en este caso como "fue un accidente que él pisara el caracol". Por ende, la situación de emisión permite responder la pregunta “¿Qué sucedió?”. Las circunstancias del movimiento excluyen su identificación como acción no porque el suceso sea o no intencional según una descripción, sino porque la descripción intencional simplemente no se aplica. Las razones del agente son irrelevantes para clasificar su movimiento corporal como acción; los accidentes son aquellos casos en los que está en disputa que alguien haya hecho algo. El mismo argumento puede usarse para los casos de Acc.3. Un accidente de este tipo es algo que sucede sin que pueda hablarse de un agente. La función predicativa corre por cuenta de la expresión "pisar", y lo que ponemos en duda es que podamos utilizarla con propiedad en una aserción como:

1. "Él pisó el caracol".

La diferencia entre llamar "acción" a un suceso y describirlo como acción parece análoga a la que existe entre nombrar y describir. En los dos casos conectamos las palabras con los sucesos (es la misma dirección de ajuste), pero al describir no se da por sentado el tipo de movimiento 
al que pertenece su movimiento físico, sino que se presupone el sentido de la expresión "Él pisó el caracol" (el onus de encaje es diferente). Nos preguntamos si el tipo de este movimiento corporal es tal que encaje con ese sentido; si pisar el caracol, dadas las circunstancias de emisión, es algo que él podría desear o conocer o explicar (Anscombe 1979, p. 220). A diferencia de otro tipo de descripciones, la descripción intencional requiere que el agente sea capaz de juzgar su acción como un todo. El adverbio funciona como un operador parentético que marca los límites de la acción (Austin 1957, p. 285). Si, por ejemplo, afirmamos:

vi. "Él pisó el caracol intencionalmente",

debemos notar que el adverbio cumple con la función de un modificador orientado al sujeto. Por lo tanto, debe parafrasearse como "fue intencional de su parte que él pisara el caracol" (Davidson 1995, p. 154). Esa descripción parece una respuesta natural a un sentido de la pregunta “¿Pretendía él pisar el caracol?”. El suceso de que él haya pisado el caracol se presenta bajo una forma en que las razones del agente lo comprometen con un determinado curso de acción. En este tipo de descripción no intentamos identificar sucesos como acciones porque el onus de encaje está puesto en la forma en que el agente concibe su acción y no en alguna característica especial del movimiento físico. La predicación recae sobre "Él pisó el caracol", pero se ajusta o no al suceso únicamente a través de las creencias y deseos del agente y no a través de las características físicas del movimiento. Tal vez Austin sugiera con su terminología que no toda descripción es identificatoria, ${ }^{19}$ y esta precisión parece iluminar las dificultades que él señala cuando se trata de describir una acción e identificarla (Austin 1957, pp. 200-201).

¿Qué hacer con el adverbio "intencionalmente" en las posiciones Acc.3 y Acc.4? Por ejemplo:

vii. "Él, intencionalmente, pisó el caracol".

Como se mostró en el caso de i y ii, esta posición señala un papel para el agente diferente al de vi. Ese aspecto faltante es el de un plan: así como una acción puede ser torpe con respecto a un plan pero no con respecto a otro, una acción puede describirse como intencional con respecto a una intención global que se pretende llevar a cabo (expresada con vi), pero no con respecto a otra. ${ }^{20}$ Por ejemplo, podemos pensar en aquellas

${ }^{19}$ Una idea que es relativamente aceptada. Para una discusión de este asunto sustentada en la bibliografía clásica véase Chateaubriand 2001 (cap. 3).

${ }^{20}$ Las semejanzas con la distinción de Searle 1999 (cap. 2) entre "intención en la acción" e "intención previa" no deben ocultar el hecho de que no me comprometo

Diánoia, vol. LX, no. 74 (mayo de 2015). 
situaciones en las que parece haber una descripción intencional que no refleja la preferencia del agente al realizar su acción o bien en las que hay una falta de control en su realización de un plan (i y ii) o bien un percance durante la realización de un plan. Todas estas posibilidades señalan una diferencia de énfasis con respecto a vi: afirmar simplemente "fue intencional de su parte pisar el caracol" como paráfrasis de vii deja de lado la circunstancia de que "pisar el caracol" no coincide con el tipo de movimiento que él había planeado aunque sea un movimiento físico controlado. Su acción, como él la vería, no debería describirse en términos de vi, sino clasificarse en términos de la ejecución de otra intención (de la intención de hacer otra cosa).

En vii deberíamos decir algo como "fue intencional de su parte pisar el caracol al ejecutar su intención de acercarse a su amigo". Sólo en este sentido la descripción intencional se toca con la pregunta por "el cómo" de la acción. No se trata de una forma de ejecución con un aspecto físico particular, sino de la ejecución o puesta en marcha de una intención global. De manera similar, podríamos parafrasear los casos i y ii como "fue torpe de su parte pisar el caracol al ejecutar su plan de alcanzar a su amigo", y podríamos parafrasear las atribuciones de accidentalidad en las posiciones Acc. 3 y Acc. 4 como "fue un accidente pisar el caracol al ejecutar su plan de alcanzar a su amigo". No cualquier suceso cuenta como un accidente en el contexto de un plan. Por ejemplo, v no presupone un plan de nadie. Todos estos casos son ejemplos del acto de habla de clasificar movimientos físicos (controlados y justificados, incontrolados con respecto a un plan o simplemente frustrados en la ejecución) con respecto a la expresión "pisar el caracol". Describir y clasificar se diferencian en su dirección de ajuste, como vimos, pero coinciden en su onus de encaje. Queremos saber de qué tipo de modelo podría ser muestra su movimiento físico, y la respuesta que se desprenderá de la discusión posterior es que su movimiento físico puede ser muestra de una intención ejecutoria (vii) o de una intención global (vi). El sentido de la aserción específica determina el sentido de la acción tal como el hablante la describe al justificarse.

Hemos conseguido traducir al lenguaje de la acción las cuatro variedades de aserción en $\mathrm{S}_{1}$ : llamar, ejemplificar, describir y clasificar. Hemos mostrado sus relaciones lógicas en términos de onus de encaje y dirección de ajuste. En la siguiente sección nos ocuparemos de cómo podemos compararlas en términos de sus respectivas negaciones.

con su teoría de la representación ni con su análisis total de la acción en términos de causas ni mucho menos con su distinción entre "presentación" y "representación". La similitud es formal, no de contenido. 


\section{Aserción, inferencia y negación}

El inferencialismo contemporáneo, por ejemplo el de Brandom 2009, nos ha enseñado a pensar el vocabulario lógico formal como una expresión de los compromisos del contenido material de la oración determinado por el uso público de nuestros conceptos comunes. Su explicación se fundamenta en las nociones de compromiso y legitimación. Aunque el sesgo de conductismo social de la propuesta y su aceptación de la idea de pensamiento fregeano resulten discutibles desde mi enfoque, parece que las cuatro variedades de aserción ya descritas sí expresan un determinado contenido en el contexto de unas prácticas de inferencia.

En 3.1 describo las prácticas que provienen de la distinción de Austin entre excusas y justificaciones. En ellas se muestra la estructura lógica del concepto de acción sin necesidad de recurrir a un vocabulario lógico. En 3.2 muestro que podemos entender el papel de la negación como la expresión de las incompatibilidades conceptuales que surgen de reglas de corrección en el contexto de esas prácticas y no necesariamente de propiedades de un dispositivo formal. Una de las mayores diferencias entre Frege y Austin reside justamente en la delimitación de la aplicación de los conceptos mediante la negación (Narboux 2011b). Intento, por lo tanto, una interpretación de la negación que le haga justicia a todas las variedades de aserción ya descritas y que sea capaz de expresar las incompatibilidades conceptuales que sugiero a lo largo de este trabajo.

\subsection{Aserción e inferencia}

"Llamar", "ejemplificar", "describir" y "clasificar" son las variedades en las que estructuramos lógicamente nuestro concepto de acción en inferencias. Las excusas y justificaciones son algunas de las prácticas argumentativas en las que hacemos explícita la fuerza específica de esas variedades. A grandes rasgos, esas prácticas se orientan por dos objetivos, o bien mostrar que hay cierta anomalía en aseverar que $x$ hizo A - una práctica atenuante- o bien mostrar lo que $x$ hizo bajo una luz más favorable (Austin 1957, p. 176) —una práctica justificativa pero posiblemente agravante-.

Las prácticas atenuantes son diversas. Por ejemplo, se puede argüir que no es verdadero decir que $x$ hizo A porque el suceso en el que $x$ se vio envuelto fue un accidente. Fue un accidente que $x$ hiciera A. En esta lectura el adverbio funciona como una indicación de que resulta inapropiado utilizar el verbo como verbo de acción. La accidentalidad del suceso nos indica una forma de poner en suspenso ese uso. En las 
prácticas argumentativas en que se utilizan oraciones como (v.) se pretende poner en duda el predicado "hizo" y mostrar que el suceso simplemente no puede describirse intencionalmente, que no tiene sentido buscar una descripción intencional. Pero tampoco tiene sentido decir que alguien no hizo algo porque no hacer A tampoco estaba ni podía estar bajo su control. El discurso de acción presupone la no accidentalidad del suceso del que se intenta predicar. Si no podemos llamar "acción" a un suceso, no podemos utilizarlo como sujeto de predicación o como tema de descripción en el discurso de la acción porque no cumple con requisitos para predicar de él o describirlo. ${ }^{21}$ Esta práctica parte de (v.) y concluye que A no es una acción (de $x$ ni de nadie) y exculpa al agente.

Una segunda práctica atenuante, si bien no necesariamente exculpatoria, consiste en mostrar un conflicto entre descripciones de la misma acción. De acuerdo con ciertas descripciones, la acción resulta excusable; según otras, reprobable. Como Davidson ha señalado, estos casos parecen involucrar problemas para la identificación de las acciones porque crean contextos oracionales referencialmente opacos (Davidson 1995, pp. 138-139). Aquí caben nuestros casos más difíciles, como (vii.). Presuponemos que el suceso no es un accidente, pero discutimos si es verdadero decir que $x$ hizo simplemente A.

Hay varios factores que podrían haber conspirado contra el agente en estas situaciones de habla: $x$ puede haber sido torpe con respecto a la ejecución de su plan de hacer A o bien puede haber sufrido un percance con respecto a ese plan o incluso puede haber cometido un lapsus en la puesta en práctica de una intención que le permite hacer A en vez de B. Es con respecto a la descripción intencional de A que el agente busca mostrar su responsabilidad y por eso la excusa puede no exculparlo. Los factores eximentes (torpeza o accidentes en la ejecución del plan) están mezclados con factores agravantes como intenciones y propósitos.

Supongamos por ejemplo que en una calurosa tarde de verano al volver al estacionamiento de su oficina una persona ve una multitud rodeando su automóvil. Ha dejado a su hijo en el asiento trasero y no sobrevivió a la hipertermia (Amaya 2013). Interrogada por la policía,

${ }^{21}$ Predicar o describir requieren la existencia de aquello que se describe o sobre lo cual se predica para no ser sinsentidos, actos "vacíos" (Austin 1975, p. 51) cuando el término descrito está en la posición del sujeto, es decir, cuando el movimiento físico que intento describir no es una acción. La influencia del trabajo de Chateaubriand en mi propuesta es evidente. pero no espero que él comparta mi análisis del discurso de la acción. 
esa persona podría contar la siguiente historia: "Esta mañana salí de mi casa con la intención de llevar el niño a la guardería. En el camino a la guardería, y sin saber cómo, vine a parar a la oficina". Su intención de hacer A (llevar al niño a la guardería, que es lo que él prefería) no fue eficiente y por eso hizo B (conducir a la oficina). B tiene una descripción intencional: no fue un accidente, ni producto de la coacción. Tampoco fue una torpeza de su parte conducir a la oficina, no con respecto a su intención de llegar a la oficina; no tuvo ningún percance en el proceso. ${ }^{22}$ Simplemente su intención de hacer A chocó en la ejecución con su intención de hacer $\mathrm{B}$. La descripción intencional es fragmentaria porque lo que él prefiere hacer no puede clasificarse como lo que él termina haciendo. Desde el punto de vista lógico la pregunta no es si él diría que dirigió el carro a la oficina (de hecho, ya admitió eso), sino si sus movimientos físicos controlados correspondieron o no a lo que él prefería, a lo que él pretendía hacer. No es verdadero decir que él simplemente condujo hacia la oficina. En esta práctica, el énfasis está puesto en la dificultad de clasificar su acción de conducir hacia la oficina como algo que él prefería y la conclusión del argumento es que él no hizo lo que pretendía hacer, sino otra cosa.

Otro tipo de práctica es la de ejemplificar un tipo de acción. Los mejores ejemplos que encuentro son las atribuciones de acción inteligente propuestas por Ryle 2005 y que parecen conectadas conceptualmente con la discusión sobre la semántica del término "real" planteada por Austin 1964 (p. 73). Cada vez que encontramos una acción de este estilo, y para Ryle todo concepto conectado con la conducta inteligente es de este estilo, podemos enseñar o corregir a alguien en la práctica correspondiente. "Bailar", "calcular", "jugar ajedrez" pueden enseñarse y aprenderse de esa forma. Una característica de esta aplicación del concepto de acción es que muestra un rasgo lógico interesante ya advertido por Grice 1988 (pp. 82-83), a saber, la aplicación de " $x$ es A" es lógicamente posterior a " $x$ es un buen A". Ser una acción de cierto tipo requiere que el propósito para ese tipo de acciones se cumpla. Como muchos de nosotros tristemente sabemos, los movimientos frenéticos o mecánicos de Julio César pueden ser un excelente contraejemplo de bailar salsa. Por esa razón es posible pasar de

"Julio César bailó salsa torpemente"

a

${ }^{22}$ Para una discusión y rechazo de cada uno de estos factores eximentes en el caso de los lapsus (slips) véase Amaya 2013.

Diánoia, vol. LX, no. 74 (mayo de 2015). 
"Julio César no bailó salsa".

En este caso, "torpemente" marca en la primera oración la incompatibilidad conceptual que expresamos mediante la negación en la segunda. A diferencia de las otras dos prácticas, ésta no funciona como eximente o agravante. Usar una oración como

"Bruto mató a César torpemente",

no cumple con la función de eximir a Bruto de la responsabilidad por haber matado a César; tampoco parece agravar su situación. Simplemente califica su forma de asesinar a César en el contexto de un determinado modelo de asesinato. Tiene sentido en boca de un criminalista reputado o de un asesino profesional. En la medida en que muchas acciones tienen propósitos relativamente impersonales (Austin 1957, pp. 285-286), podemos evaluarlas desde este punto de vista. Sin embargo, puede cuestionarse que este esquema de inferencia funcione con cualquier verbo de acción o con cualquier concepto, incluso con cualquier tipo de torpeza. No creo que muchos de nosotros estemos dispuestos a decir, por ejemplo, que de

"Babieca es un caballo torpe"

podamos inferir

"Babieca no es un caballo".

Por último, la práctica conectada con vi es agravante y justificativa. Con ella queremos hacer manifiestas nuestras razones para hacer lo que hicimos, independientemente de si son buenas o malas objetivamente. A diferencia de vii, la fuerza de la aserción está puesta en otorgarle cierta unidad a nuestra acción. Es verdad que el atribulado padre del ejemplo del lapsus ha realizado una serie de movimientos físicos —descritos como "conducir al trabajo" - que podrían clasificarse como intencionales dadas las circunstancias. Lo que no es verdad, o al menos es discutible, es que él afirme que pretendía conducir directo al trabajo cuando salió de su casa. Tiene sentido decir que él condujo directamente al trabajo pero que no pretendía hacerlo, porque conducir al trabajo trajo como consecuencia la muerte de su hijo, algo que él nunca pretendió hacer. $\mathrm{Si}$, como he argumentado en 2.2 , la posición del modificador adverbial determina un sentido, vi es justamente el sentido que plantea una intención que puede o no ejecutarse. Es una respuesta de tipo sí/no a una pregunta planteada por una intención, a saber,

“¿Pretendías conducir directo al trabajo cuando saliste de casa?”. 
A lo que él podría contestar:

"No, pretendía llevar a mi hijo a la guardería".

Así, es posible afirmar que en los lapsus el agente considera verdadera la clasificación del tipo vii, mientras que a la descripción del tipo vi la considera falsa, dadas las razones que él tenía para hacer lo que hizo. El agente no se comprometería con ese curso de acción, dado todo lo que creía y quería al salir de su casa. Cuando se utiliza vi en una situación de habla el contenido de la acción, tal como la concibió el agente, es relevante y el enunciado presenta ese contenido de una manera estructurada por las razones del agente. Este tipo de práctica se conecta con la necesidad de dotar de contenido a una acción a partir de las razones que la justifican, lo que no quiere decir que no haya otras formas de lograrlo. La otra cara de la moneda son los ejemplos de acciones que tienen un contenido general (un propósito) independientemente de las intenciones del agente. Ambas variedades de aserción, ejemplificar y describir, presentan la acción completamente, sea desde su apariencia física, sea desde las razones que la motivaron. La práctica justificativa concluye con una descripción de lo que el agente pretendía hacer a partir de sus posibles consecuencias. Esa forma de expresar el contenido determina una característica lógica conectada con la contradicción, como veremos.

\subsection{Dicotomía, contrariedad y contradicción}

El uso de la negación en Austin presupone una crítica a la ideología que subyace en la lógica formal. Esa ideología parte de la tesis que se acepta sin discutir de que los criterios de evaluación no son ellos mismos evaluables. En el caso de las aserciones que incluyen el concepto de acción esto puede interpretarse como si todo aquello que puede considerarse una acción tuviera una sola forma de evaluarse, la de una pregunta de tipo sí/no formulada por una intención (Narboux 2011b, p. 214); es decir, como si solamente fuera posible una evaluación en términos de la práctica de justificación recién descrita. Pero hay muchas formas en que una acción puede evaluarse, muchas maneras en que puede salir mal, como lo muestran las otras tres prácticas. Esas formas de salir mal nos conducen directamente a preguntarnos si hay una sola forma de evaluar la aserción a efectos de que un determinado movimiento físico sea una acción. La respuesta a esta pregunta es negativa. Todas las posibilidades presentes en las aserciones sobre la acción señalan una importante diferencia con Frege, la imposibilidad de aplicar o no apli-

Diánoia, vol. LX, no. 74 (mayo de 2015). 
car un concepto siempre y para todo propósito de una forma definida. El concepto de acción no responde a una lógica dicotómica.

Algunas veces evaluamos o criticamos movimientos físicos como acciones de diferentes maneras, con diferentes objetivos y en diversos grados. A veces una aserción sobre la acción sin negación ni términos privativos excluye otra aserción sobre la acción, otras veces la negación adecuada para excluir una aserción como correcta es metalingüística en el sentido de Horn 2001, y Horn y Speranza 2010, y señala que la situación de habla no es exactamente compatible con la aserción. A veces la negación señala oraciones contrarias pero no contradictorias porque el espacio lógico es abierto (multidimensional).

Consideremos la ejemplificación. En este caso el criterio de evaluación es también un criterio de ser: un movimiento torpe de acuerdo con un modelo no es un determinado tipo de acción. Cuando afirmo iii o iv descarto una situación de habla, aquella en que resulta correcto decir que él pisó el caracol y, por ello, afirmo que es falso que él haya pisado el caracol. Por esa razón puedo pasar de iii o iv a

n.e. "Él no pisó el caracol".

El adverbio de modo señala que su movimiento físico no cuenta como "pisar el caracol" en el concurso de pisar caracoles con la punta de los pies. Si ese parámetro de evaluación está dado, entonces n.e. y e. expresan pensamientos contradictorios: ambas aserciones no pueden ser verdaderas ni falsas en la misma situación de habla. Dado un parámetro social, o bien su pisotón del caracol fue torpe, o bien no lo fue. De eso depende el concurso. Pero no podemos inferir a partir de la negación de iii que él haya pisado el caracol debido a que la negación de iii es metalingüística. Si es correcto decir

"Él no pisó el caracol torpemente",

únicamente podemos inferir que lo pisó de otra manera (por accidente, por error o hábilmente), sólo excluimos una forma de crítica aplicable a su pisotón del caracol sin determinar qué otro tipo de crítica resulta adecuada.

Algunas veces el criterio de ser es un criterio de evaluación. Cuando la acción no se completa o lleva a cabo de cierta manera (como respuesta a un plan), puede resultar incorrecto aseverar que el agente llanamente hizo A. Por ejemplo, cuando pisar el caracol puede ser visto como un lapsus resulta extraño aseverar simplemente c. Afirmar que él pisó simplemente el caracol es incorrecto porque él pisó el caracol mientras se acercaba a su amigo. Hablar del pisotón sin mencionar lo que él 
pretendía hacer es inadecuado en esa situación de habla. Clasificar sus movimientos corporales como intencionales requiere entonces aclarar que son intencionales en la consecución de un fin. Lo interesante de este caso es que las condiciones de falsedad no requieren una negación sintácticamente marcada. Por ejemplo, si es correcto afirmar

ac.c. "Él, por accidente, pisó el caracol",

entonces es incorrecto afirmar vii; ambas no pueden ser verdaderas. Si ac.c. es verdadero, es correcto decir que él pisó el caracol como resultado de un imprevisto que impidió responder a sus verdaderas intenciones. Resulta incorrecto decir que él pisó el caracol como parte de un plan; luego es incorrecto afirmar vii y, por ende, vii es falso. Pero lo que puede clasificarse como accidente con respecto a un plan, podría clasificarse como torpe o como intencional de acuerdo con otro. Ningún suceso es torpe o accidental per se en esta especie de aserción. Por esta razón, las oraciones de acción en su uso clasificatorio no admiten una sola contradictoria, pero sí muchas contrarias.

Por ejemplo, tomemos:

n.c. "Él no pisó el caracol"

y comparemos n.c. con:

n.vii. "Él, intencionalmente, no pisó el caracol”.

n.ac.c. "Él, por accidente, no pisó el caracol".

n.t.c. "Él, torpemente, no pisó el caracol".

En cada uno de estos casos resulta incorrecto afirmar sin más que él no pisó el caracol. Por eso se requiere el uso de adverbios como "simplemente" o "exactamente" en la explicitación de las condiciones de corrección (Narboux 2011b, p. 226). Cuando digo que no es correcto decir simplemente que él no pisó el caracol, afirmo que es falso que él, sin más, no haya pisado el caracol. Él no lo pisó o pasó por alto pisarlo en la ejecución de un plan de no pisarlo (n.vii); o bien no lo pisó por falta de control en la ejecución de un plan para pisarlo (n.t.c) o bien por un imprevisto en la puesta en marcha de su plan de pisarlo (n.ac.c).

$\mathrm{Si}$ vii y ac.c fueran contradictorias, no podrían ser ambas falsas. Si él no consigue pisar el caracol por falta de control y no por un elemento extraño a su plan de no pisarlo, es incorrecto afirmar n.ac.c, pero también n.vii porque, por hipótesis, su plan no se cumple. Luego, ac.c y vii. son contrarias, en línea con las conclusiones de Austin sobre las aserciones clasificatorias (Austin 1957, p. 152). Este comportamiento 
lógico trae algunas consecuencias para algunos temas discutidos en filosofía de la acción.

Tomemos el caso del padre desafortunado que intencionalmente conduce directo al trabajo en vez de dejar a su hijo en la guardería. Hay una clasificación de sus movimientos físicos controlados que encaja con el sentido de la expresión "conducir directo al trabajo". Pero no podemos encontrar ninguna clasificación que encaje con el sentido de la expresión "no dejar el niño en la guardería", porque dicha expresión no puede identificar movimiento alguno. ¿Cuáles de sus movimientos concuerdan con su sentido expresión? ¿Todos los que realizó de camino a su oficina? ¿Los de hace una semana? ¿Los posteriores al parto del niño? Las preguntas señalan lo absurdo de identificar A como no B, los movimientos físicos del padre como "no dejar el niño en la guardería". Para usar un lema austiniano: "identificar como no es un sinsentido por no identificar." (Austin 1957, p. 153). ${ }^{23}$ El fin de la acción del padre era, justamente, dejar al niño en la guardería. Esa clasificación de sus movimientos físicos falla: él no los identifica como una parte de su plan de dejar al niño en la guardería. A diferencia de la ejemplificación, en la clasificación la verdad de la oración de acción sin modificador no se ve afectada — como se puede apreciar con n.vii, n.ac.c y ntc- - La posibilidad de identificar el movimiento físico como la ejecución de un plan, el plan que establece su onus de encaje, parece ponerse en entredicho. La incompatibilidad se produce entre ese y otros de los planes posibles.

En contraste, una característica de las aserciones que son descripciones es que admiten contradictorios. Cuando alguien afirma vi, no puede afirmar al mismo tiempo:

n.vi. "Él no pisó el caracol intencionalmente".

Mientras que vi es verdadero siempre que sea correcto decir que él pretendía pisar el caracol, n.vi es verdadero siempre que sea correcto decir que él no pretendía pisar el caracol. Si él pretendió pisar el caracol, si el contenido total de su acción tal como él la vio era que él pisara el caracol, no pudo no haber pretendido pisar el caracol (vi y n.vi) no pueden ser ambas verdaderas), aunque ambas presuponen que él pisó el caracol. La práctica de justificación nos puede dar una buena pista de por qué las descripciones de acción funcionan lógicamente así.

${ }^{23}$ En vena austiniana yo no diría que bajo la descripción de "no dejar al niño en la guardería de camino a su oficina" la acción del padre no es intencional (Amaya 2013, p. 561). Diría (i) que ésa no es una descripción, sino una clasificación y (ii) que es una clasificación fallida, sin sentido. Para una discusión sistemática de la identificación negativa, véase Narboux 2011a.

Diánoia, vol. LX, no. 74 (mayo de 2015). 
El agente intenta presentar su acción como él la concibió y esa presentación excluye descripciones parciales que la fragmentan. Al darle unidad, el agente hace algo más: se compromete con ese suceso como su acción, con las consecuencias que ella pueda traer (Austin 1957, p. 285). No puede afirmar que fue y no fue intencional de su parte pisar el caracol porque en ese caso no sería capaz de justificar las razones que lo llevaron a pisarlo. La contradicción entre descripciones señala la imposibilidad de que un agente justifique su acción, es decir, la imposibilidad de que su acción tenga un contenido para él. Lo curioso del caso es que la inconsistencia no se da porque el contenido fregeano sea contradictorio. Es una contradicción entre tener razones para hacer algo y no tenerlas, entre concebir una acción bajo una determinada luz y no concebirla en absoluto. Decir que no fue intencional de su parte pisar el caracol o que no pretendía pisar el caracol es señalar que el agente no tiene una historia que contar sobre el pisotón del caracol aunque éste haya sucedido. Decir que fue intencional es comenzar a contar la historia de por qué sucedió, de acuerdo con el agente.

Las aserciones en las que llamamos "acciones" a un ciertos sucesos comparten características con las descripciones, clasificaciones y ejemplificaciones. Al igual que las descripciones, presentan el suceso de una manera unitaria aunque, análogamente a las ejemplificaciones, sin referencia al supuesto agente. Los accidentes, como los ejemplos, no requieren la evaluación del agente para ser acciones, pero en los accidentes el criterio de evaluación no requiere un conjunto de modelos de acción. Son casos en que movemos nuestros cuerpos, pero no podemos dejarle el resto a la naturaleza porque nos falla. Los accidentes nos muestran que somos físicamente limitados y que esos límites no dependen de nuestra habilidad, racionalidad o adiestramiento. En los accidentes el criterio de evaluación no es un criterio de ser. Por ejemplo,

n.v. "Él no pisó el caracol por accidente"

permite apreciar las similitudes entre diferentes formas de identificación. Como en el caso de la clasificación, aquí tampoco tiene sentido llamar a un suceso "no accidente". Lo que decimos al afirmar n.v. es que no podemos identificar un suceso como accidente. Al ser incorrecto decir que él pisó el caracol por accidente, en realidad señalo un abanico de aserciones correctas. Sería correcto decir que él pisó el caracol torpemente o que él pisó el caracol por error o intencionalmente y estas aserciones se excluyen (Austin 1957, pp. 176, 185, 279). Pero de n.v. no podemos pasar sin otro argumento a la conclusión de que él pisó el caracol intencionalmente; no podemos descartar las otras aserciones 
sin que la situación de habla cambie. Que un suceso no suceda accidentalmente no implica que sea una acción pretendida por un agente en el sentido fuerte de la práctica justificativa. Sólo quiere decir que es un movimiento que, por sus características físicas, vale como candidato posible para transformarse en una acción, y no que alguien lo haya concebido y ejecutado como tal. ${ }^{24}$

\section{Conclusiones}

No hay señales claras de cómo pretendía Austin compaginar el sutil modelo de aserción $\mathrm{S}_{1}$ con sus agudas intuiciones en filosofía de la acción. A lo largo de este trabajo he indicado una forma posible de extender sus resultados sobre la dirección de ajuste y el onus de encaje para que sus tesis sobre la modificación adverbial puedan recibir el tratamiento sistemático que merecen. He sostenido en la segunda sección que las cuatro variedades más complejas de aserción (llamar, ejemplificar, describir y clasificar) permiten entender el origen de algunos problemas básicos en filosofía de la acción y he resaltado la función que cumplen algunos adverbios en ese proceso. Ese papel se hace explícito una vez que la negación entra en escena, tal como predijo Austin en su trabajo sobre excusas, porque la negación es una forma de indicar incompatibilidades conceptuales que surgen de una anomalía en la situación de habla. Sólo si prestamos atención a las situaciones en las que necesitamos excusar, justificar o ejemplificar nuestras acciones podemos entender esas anomalías. He argumentado en la tercera sección que las cuatro variedades de aserción presentadas en la segunda sección pueden ponerse al servicio de diferentes tipos de prácticas de inferencia (atenuantes, agravantes o ejemplarizantes) en las que los adverbios expresan cómo articulamos en nuestra lengua las incompatibilidades y exclusiones propias de nuestro concepto de acción.*

${ }^{24}$ No excluyo la posibilidad de que una práctica pueda mezclarse con otra, de que en una situación de habla un tipo de contenido asertivo no sea tan claramente distinguible de otro como un lógico lo desearía. Simplemente señalo cuatro variedades generales (no las únicas, pero si algunas de las más discutidas) en que puede estructurarse ese posible contenido.

*Este trabajo hace parte del proyecto de investigación Lenguaje y acción. Fundamentos de una semántica multidimensional, financiado por la Universidad de los Andes, Colombia. En versiones parciales fue presentado en Bogotá, Buenos Aires y Santiago de Chile y como requisito de ingreso a la Sociedad Colombiana de Filosofía. Agradezco a Carlos Patarroyo y Carlos Cardona de la Universidad del Rosario, a María del Rosario Acosta de la Universidad de los Andes, a José Tomás Alvarado de la Pontificia Universidad Católica de Chile y a las evaluaciones anónimas de Diánoia 


\section{BIBLIOGRAFÍA}

Amaya, S., 2013, "Slips", Noûs, vol. 47, no. 3, pp. 559-576.

Annas, J., 1976, "Davidson and Anscombe on 'The Same Action"', Mind, vol. 85, no. 338 , pp. $251-257$.

Anscombe, G., 1979, "Under a Description”, Noûs, vol. 13, no. 2, pp. 219-233. Austin, J.L., 1940, "The Meaning of a Word", en Austin 1979, pp. 55-75.

_ 1953, "How to Talk. Some Simple Ways", en Austin 1979, pp. 134-153.

, 1957, "A Plea for Excuses", en Austin 1979, pp. 175-204.

—_, 1966, "Three Ways of Spilling Ink", en Austin 1979, pp. 272-287.

— 1964, Sense and Sensibilia, G.J. Warnock (ed.), Oxford University Press, Nueva York.

—, 1975, How to Do Things With Words, J.O. Urmson y M. Sbisá (eds.), Harvard University Press, Cambridge.

— 1979, Philosophical Papers, J.O. Urmson y G.J. Warnock (eds.), Oxford University Press, Oxford.

Barker, S.J., 2004, Renewing Meaning. A Speech-Act Theoretic Approach, Oxford University Press, Oxford.

—_, 2007, Global Expressivism: Language Agency without Semantics, Reality Without Metaphysics, University of Nottingham eprints, Nottingham, disponible en <http://eprints.nottingham.ac.uk/696/1/BOOKGE.pdf>, consultado por última vez el 12 de junio de 2013 .

Barrero, T., 2013, "Acción y construcción lógica", Crítica, vol. 45, no. 133, pp. 3-26.

Brandom, R.B., 2009, Hacerlo explícito. Razonamiento, representación y compromiso discursivo, trad. A. Ackermann y J. Rosselló, Herder, Barcelona.

Chateaubriand, O., 2001, Logical Forms. Part I. Truth and Description, col. CLE, UNICAMP, Campinas.

Chisholm, R.M., 1969, “Austin's Philosophical Papers", en K.T. Fann, pp. 101126.

Davidson, D., 1995, Ensayos sobre acciones y sucesos, trad. O. Hansberg, J.A. Robles y M. Valdés, Crítica-Instituto de Investigaciones Filosóficas-UNAM, Barcelona/México.

Fann, K.T., 1969, Symposium on J.L. Austin, Routledge \& Kegan Paul, Londres. Forguson, L., 1973, "Locutionary and Illocutionary Acts", en Essays on J.L. Austin, Oxford University Press, Oxford, pp. 160-185.

Frápolli, M.J., 2013, The Nature of Truth. An Update Account of the Meaning of Truth Ascriptions, Springer, Dordrecht.

Frege, G., 1998, Ensayos sobre semántica y filosofía de la lógica, trad. y ed. L.M. Valdés, Tecnos, Madrid.

por sus críticas y comentarios. También me beneficié de una conferencia de Jaime Ramos de la Universidad Nacional de Colombia en el IV Congreso colombiano de filosofía en Manizales. 
García Suárez, A., 1975a, "Presentación", en J.L. Austin, Ensayos Filosóficos, J.O. Urmson y G.J. Warnock (eds.), trad. A. García Suárez, Revista de Occidente, Madrid, pp. 11-28.

Grice, H., 1986, "Actions and Events", Pacific Philosophical Quarterly, vol. 67, no. 1, pp. 1-35.

-, 1988, "Reply to Richards", en R.E. Grandy y R. Warner (comps.), Philosophical Grounds of Rationality. Intentions, Categories, Ends, Clarendon Press, Oxford, pp. 45-106.

Horn, L.R., 2001, A Natural History of Negation, CSLI Publications, Stanford.

Horn, L.R. \& J. Speranza, 2010, "A Brief History of Negation”, Journal of Applied Logic, vol. 8, no. 3, pp. 277-301.

Lepore, E. \& K. Ludwig, 2007, Donald Davidson's Truth-Theoretic Semantics, Oxford University Press, Oxford.

Narboux, J.-P., 2011a, "The Logical Fabric of Assertions. Some Lessons from Austin's 'How to Talk. Some Simple Ways'", en S. Laugier \& C. Al-Saleh (comps.), John L. Austin et la philosophie du langage ordinaire, Georg Olms Verlag, Hildesheim, pp. 183-230.

—_ 2011b, “There's many a Slip between Cup and Lip': Dimension and Negation in Austin", en M. Gustafsson y R. Sørli (comps.), The Philosophy of J.L. Austin, Oxford University Press, Oxford/Nueva York, pp. 204-240.

Parsons, T., 1990, Events in the Semantics of English, The MIT Press, Cambridge. Ryle, G., 2005, El concepto de lo mental, trad. E. Rabossi, Paidós, Barcelona.

Searle, J.R., 1969, "Assertions and Aberrations", en K.T. Fann, pp. 205-218.

-, 1973, "Austin on Locutionary and Illocutionary Acts", en Essays on J.L. Austin, Oxford University Press, Oxford, pp. 141-159.

- 1979, Expression and Meaning, Cambridge University Press, Cambridge, Massachusetts.

__ 1999, Intentionality, Cambridge University Press, Cambridge, Massachusetts.

Strawson, P., 1973, "Austin and 'Locutionary Meaning'", en Essays on J.L. Austin, Oxford University Press, Oxford, pp. 46-68.

Taylor, B., 1985, Modes of Occurrence, Basil-Blackwell, Oxford.

Warnock, G., 1989, J.L. Austin, Routledge, Londres.

Recibido el 5 de diciembre de 2014; aceptado el 6 de marzo de 2015. 